\title{
Difference between even- and odd-numbered cycles in the predictability of solar activity and prediction of the amplitude of cycle 25
}

\author{
A. Yoshida \\ Shizuoka University, 836, Ohya, Suruga-ku, Shizuoka, 422-8529, Japan \\ Correspondence to: A. Yoshida (akio.yoshi@nifty.com)
}

Received: 23 January 2014 - Revised: 24 April 2014 - Accepted: 17 July 2014 - Published: 25 August 2014

\begin{abstract}
It was shown previously that the sunspot number (SSN) at a point 3 years before the minimum is well correlated with the maximum SSN of the succeeding cycle, and a better correlation is obtained when the maximum SSN is replaced by the average SSN over a cycle for which the average SSN is calculated by dividing cycles at a point 3 years before the minimum (Yoshida and Yamagishi, 2010; Yoshida and Sayre, 2012). Following these findings, we demonstrate in this paper that the correlation between the SSN 3 years before the minimum and the amplitude of the coming cycle differs significantly between even-numbered and oddnumbered cycles: the correlation is much better for evennumbered cycles. Further, it is shown that the amplitude of even-numbered cycles is strongly correlated with that of the succeeding odd-numbered cycles, while the correlation between amplitudes of odd-numbered cycles and those of succeeding even-numbered cycles is very poor. Using the excellent correlations, we estimate the maximum SSN of the current cycle 24 at 81.3 and predict the maximum SSN of cycle 25 to be $115.4 \pm 11.9$. It is of note, however, that a peak of the SSN has been observed in February 2012 and the peak value 66.9 is considerably smaller than the estimated maximum SSN of cycle 24 . We conjecture that the second higher peak of the SSN may appear.
\end{abstract}

Keywords. Solar physics, astrophysics, and astronomy (general or miscellaneous)

\section{Introduction}

Knowing solar activity in advance is an important issue not only for space-weather researchers who are in charge of giving a warning to astronauts operating in outer space but also for solar physicists who study the solar-dynamo theory and state administrators who are responsible for making spaceexploring plans. Nowadays, climatologists also have strong interests in the change of solar activity, if any sign indicating approach of a Little Ice Age is observed. Consequently, a large number of studies have been carried out trying to predict the amplitude of coming solar cycles. Since the underlying key factor that brings about the around 11-year-period variation of solar activity is still not elucidated, most of the studies are inevitably empirical ones based on various kinds of proxies that show relatedness to the amplitude of the coming cycle (e.g., Pesnell, 2008).

Among well-known proxies are counted global geomagnetic disturbance, especially its strength in the declining phase of the solar cycle (e.g., Ohl, 1966; Hathaway and Wilson, 2006; Kane, 2007), intensity of the polar magnetic field of the sun (Schatten, 2005; Svalgaard et al., 2005), and the meridional surface flow of the sun which is surmised to relate to solar dynamo (Hathaway et al., 2003, 2004; Dikpati et al., 2006; Cameron and Schussler, 2007a). Above all, the most popular index may be the sunspot number (SSN), and it is considered fundamental to study the predictability of future solar activity based on the change of the SSN, for the around 11-year-period variation of solar activity is usually represented by the change of the SSN and the sunspot is characterized by its strong magnetic field that is supposed to play an essential role in solar dynamo. It is as well expected that any regularity found out in the change of the SSN will supply us with clues to understand the mechanism of solar dynamo.

In our previous paper (Yoshida and Yamagishi, 2010), we showed that the SSNs at points 1, 2, 3, 4, and 5 years before the minimum or the minimum SSN are correlated with the maximum SSN of the succeeding cycle, and that the SSN 
(a)

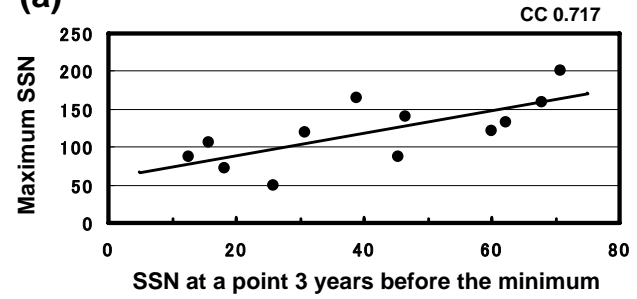

(c)

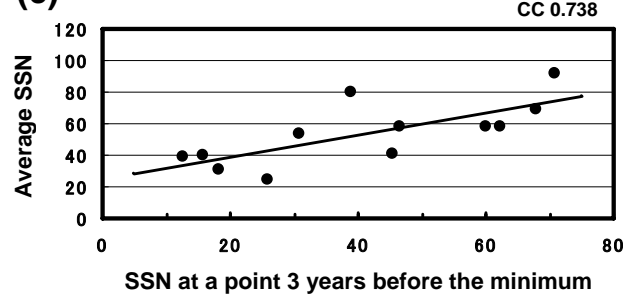

(b)

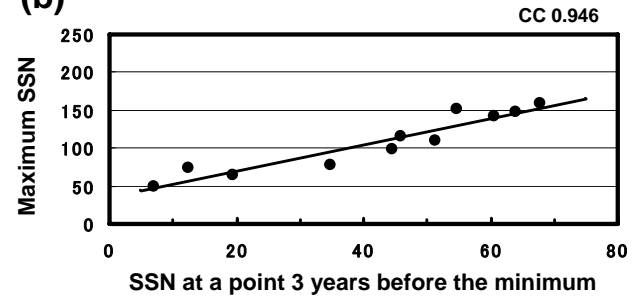

(d)

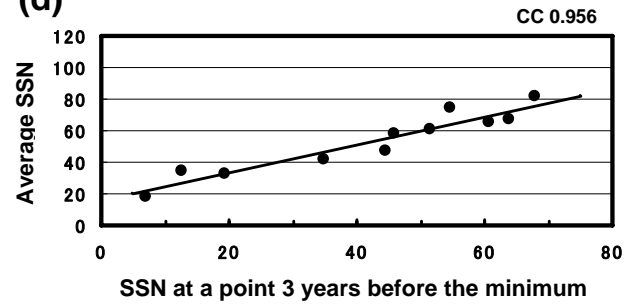

Figure 1. (a) Scatterplot of maximum SSN versus SSN 3 years before the minimum for odd-numbered cycles. (b) Same as panel (a) but for even-numbered cycles. The correlation coefficient and its lower and upper limits of the $95 \%$ confidence interval are $0.717,0.243$ and 0.921 for the former case, while those for the latter case are 0.946, 0.800 and 0.986, respectively. (c) Scatterplot of average SSN versus SSN 3 years before the minimum for odd-numbered cycles. (d) Same as panel (c) but for even-numbered cycles. The correlation coefficient and its lower and upper limits of the $95 \%$ confidence interval are $0.738,0.284$ and 0.915 for the former case, while those for the latter case are $0.956,0.833$ and 0.989 , respectively.

at a point 3 years before the minimum exhibits the strongest correlation. Yoshida and Sayre (2012), then, showed that a better correlation is obtained when the average SSN over a cycle is taken instead of the maximum SSN, and that the correlation coefficient becomes the largest when the average SSN is calculated by dividing cycles at a point 3 years before the minimum. They also showed that the correlation between the average SSN and the maximum SSN is also strongest for the average SSN thus calculated. Based on these findings, Yoshida and Sayre (2012) proposed that the average SSN is better than the maximum SSN as the representative of the amplitude of solar activity, and that a point 3 years before the minimum is the most appropriate point to define cycle's start and end.

In this paper, we demonstrate that the correlation between the amplitude (the average or the maximum SSN) and the SSN at a point 3 years before the minimum differs significantly depending on whether the succeeding cycle is an even- or an odd-numbered cycle: the correlation for evennumbered cycles is incomparably better than the correlation for odd-numbered cycles. Using the excellent correlations, we first estimate the average SSN of the current cycle 24, and then, predict the maximum SSN of cycle 25 .

\section{Difference in the predictability between even- and odd-numbered cycles}

Figure 1a and $\mathrm{b}$ show scatterplots between the maximum SSN and the SSN at a point 3 years before the minimum for odd-numbered cycles and for even-numbered cycles, respectively. The monthly smoothed SSN data are obtained from the Solar Influence Data Analysis Center (http://www. sidc.be/sunspot-data/). The correlation coefficient for oddnumbered cycles is 0.717 , while that for even-numbered cycles is 0.946 . The $P$ value in the null hypothesis test for the former case is 0.00868 , while that for the latter case is $1.085 \times 10^{-5}$, respectively, indicating that the correlation for even-numbered cycles is accepted at a very high significance level. Figure 1c and d are the same as Fig. 1a and b except that the average SSN is taken instead of the maximum SSN. In this scatterplot as well, it is seen that the correlation for even-numbered cycles is much better, giving the correlation coefficient of 0.956 against that of 0.738 for odd-numbered cycles. The $P$ value for odd-numbered cycles is 0.00618 , while that for even-numbered cycles is $4.511 \times 10^{-6}$, respectively, showing as well that the correlation for the latter case is highly significant. Here, it is to be noted that the average SSN over a cycle is calculated by dividing solar cycles at a point 3 years before the minimum (Yoshida and Sayre, 2012). The average SSN thus calculated is most strongly correlated with the SSN at the dividing point not only for evennumbered cycles but also for odd-numbered cycles.

A tendency has been long known that the larger the rate of increase of the SSN in the rising phase, the larger the maximum SSN of the cycle (Waldmeier, 1935). Thompson (1988) showed that the SSN at the point 24 months after the minimum is well correlated with the maximum SSN. We have found that, among the SSN at points 1, 2, 3, and 4 years after the minimum, the SSN at 3 years after the minimum is best correlated with the amplitude of that cycle. Figure $2 \mathrm{a}$ and $\mathrm{b}$ show scatterplots between the maximum SSN and the SSN 

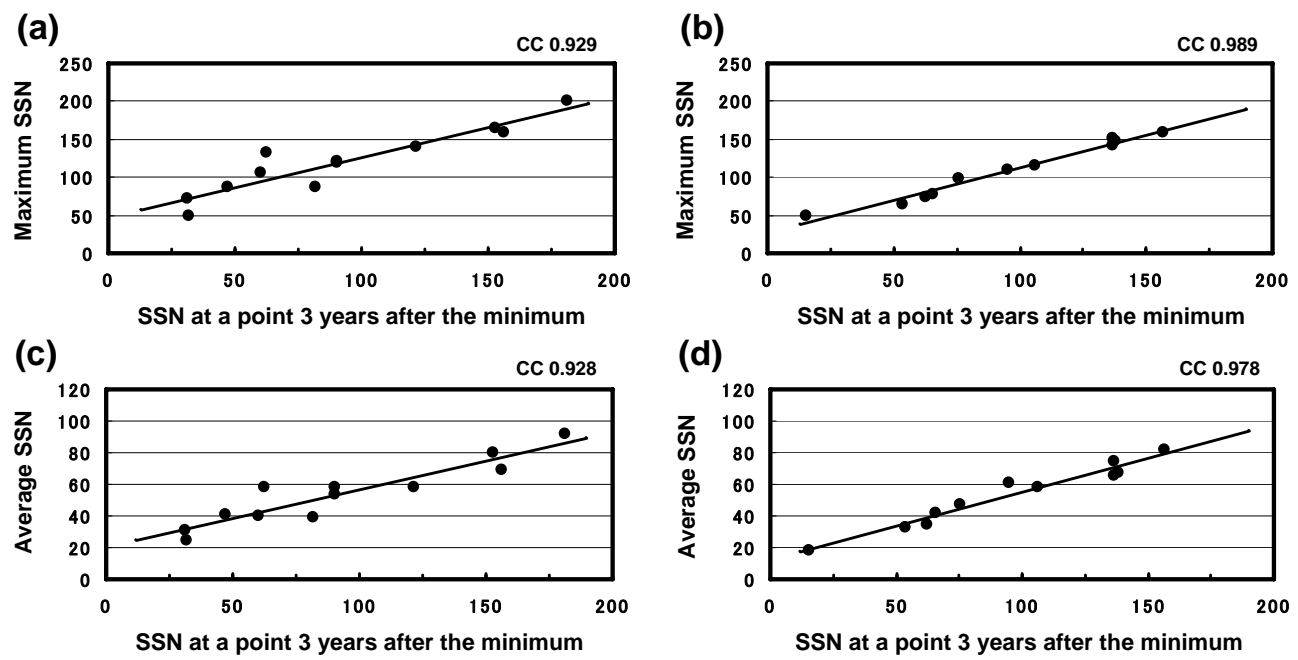

Figure 2. (a) Scatterplot of maximum SSN versus SSN 3 years after the minimum for odd-numbered cycles. (b) Same as panel (a) but for even-numbered cycles. The correlation coefficient and its lower and upper limits of the $95 \%$ confidence interval are $0.929,0.762$ and 0.980 for the former case, while those for the latter case are 0.988, 0.955 and 0.997 , respectively. (c) Scatterplot of average SSN versus SSN 3 years after the minimum for odd-numbered cycles. (d) Same as panel (c) but for even-numbered cycles. The correlation coefficient and its lower and upper limits of the $95 \%$ confidence interval are $0.928,0.759$ and 0.980 for the former case, while those for the latter case are 0.978 , 0.914 and 0.994 , respectively.

at a point 3 years after the minimum for odd-numbered cycles and for even-numbered cycles, respectively. It is seen that the correlation for even-numbered cycles (Fig. 2b) is very strong, carrying a correlation coefficient of 0.989 . Although the scatterplot for odd-numbered cycles is a bit more dispersive, the correlation is also strong, giving a correlation coefficient of 0.929 . The $P$ value for even-numbered cycles is $1.051 \times 10^{-8}$ and that for odd-numbered cycles is $1.231 \times 10^{-5}$, respectively, indicating that both of the correlations are accepted at a very high significance level. Figure $2 \mathrm{c}$ and $\mathrm{d}$ are the same as Fig. 2a and b except that the average SSN is taken instead of the maximum SSN. The correlation coefficient for odd-numbered cycles is 0.928 , while that for even-numbered cycles is 0.978 , and the $P$ value is $1.309 \times 10^{-5}$ and $2.015 \times 10^{-7}$ for odd-numbered and evennumbered cycles, respectively. Therefore, it can be said that the so-called Waldmeier effect, though it is a bit clearer for even-numbered cycles, is well recognized for both evennumbered and odd-numbered cycles. It is of note here that, in the correlation between the average SSN and the SSN at a point 3 years after the minimum, the best correlation is obtained when the average is calculated by dividing cycles at a point 3 years before the minimum, not at a point 3 years after. This fact supports our previously presented idea that a point 3 years before the minimum is the most appropriate point to define the beginning and end of the solar cycle (Yoshida and Sayre, 2012).

In summarizing the above-described results, we would like to emphasize that significant difference seems to exist in the predictability of the amplitude of the coming cycle between even-numbered and odd-numbered cycles: the amplitude of even-numbered cycles is predicted with a higher precision than that of odd-numbered cycles.

Cameron and Schussler (2007a, b, 2008) presented an idea that most well-known correlations between precursors and following cycle amplitude are explained by cycle asymmetry and cycle overlapping, where the Waldmeier effect, the larger the rate of increase of the SSN in the early stage of cycle, the larger the amplitude of the cycle, plays an essential role. If this is the case, the solar cycle amplitudes may constitute a purely random sequence whereby the amplitude is decided only by how fast the SSN increases from minimum (Cameron and Schussler, 2008). Our finding that correlations between the average SSN over a cycle and either of the SSN at a point 3 years before and 3 years after the minimum become strongest when the average is calculated by dividing cycles at a point 3 years before the minimum (Yoshida and Sayre, 2012) may be considered to support the idea. However, we think something more might be hidden in the observation of the significant difference in the correlations between even-numbered and odd-numbered cycles. The observation that the amplitude of even-numbered cycles is well correlated with the amplitude of the succeeding oddnumbered cycles (see Fig. 4) also seems to suggest existence of a kind of inter-cycle relation.

On the other hand, Dikpati et al. (2008) found that what they call the "Waldmeier effect", i.e., the correlation between cycle peak and the time from minimum to reach that peak, is not seen in sunspot area data, though it is seen in the SSN, especially for even-numbered cycles. They further studied the "Waldmeier effect" by splitting the sunspot area data 

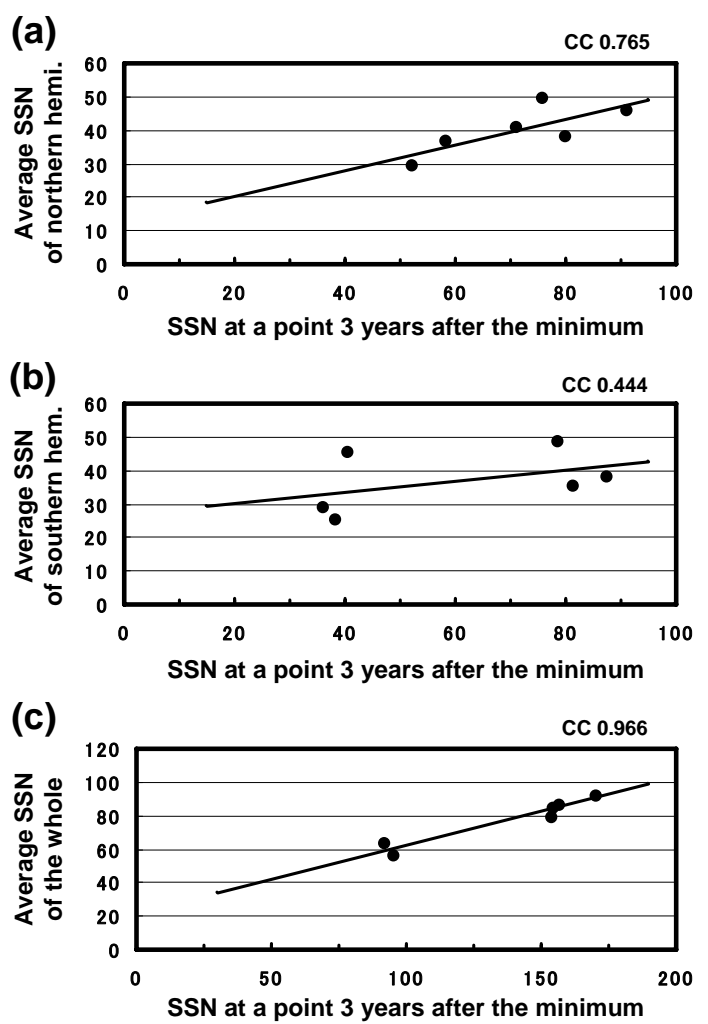

Figure 3. (a) Scatterplot of average SSN versus SSN 3 years after the minimum for the data of Northern Hemisphere. (b) Same as panel (a) but for the data of Southern Hemisphere. (c) Same as (a) but for the data of the whole sphere. The correlation coefficient and its lower and upper limits of the $95 \%$ confidence interval are 0.764 , -0.123 and 0.972 for the case (a), $0.444,-0.574$ and 0.923 for the case (b), and $0.966,0.717$ and 0.996 for the case (c), respectively. In these plots we used the SSN data during cycles 18 to 23 obtained by the National Astronomical Observatory of Japan by obtaining 13-month averages.

into Northern and Southern hemispheres, and found that the "Waldmeier effect" is not seen for either data.

We examined if the Waldmeier effect, in the sense of that by Cameron and Schussler (2008), is recognized in the hemisphere SSN data. Figure 3a, b, and c show scatterplots of average SSN versus SSN 3 years after the minimum for the SSN of Northern and Southern hemispheres and that of the whole sphere, respectively, where average SSNs are calculated by dividing the cycle changes at a point 3 years before each of the minima. In the analysis we used the SSN data during cycles 18 to 23 obtained by the National Astronomical Observatory of Japan by obtaining 13-month averages. It is apparently seen that the correlation for the SSN of the whole sphere is strongest, that for the Northern Hemisphere SSN is the next, and that for the Southern Hemisphere SSN is weakest. The correlation coefficient is $0.765,0.444$ and 0.966 for the Northern Hemisphere SSN, for the Southern Hemisphere SSN, and for the SSN of the whole sphere, respectively, and the $P$ value for each of the correlations is $0.0765,0.378$ and 0.002 . Therefore, only the correlation (the Waldmeier effect) is not denied for the SSN of the whole sphere at a confidence level of $1 \%$.

Having made separate simulations of sunspot cycles for Northern and Southern hemispheres by using the fluxtransport dynamo prediction scheme (Dikpati et al., 2006), Dikpati et al. (2007) found that the skill level was only slightly lowered. Belucz and Dikpati (2013), who conducted simulations of solar cycles by changing properties of meridional circulation only in the south, obtained results that the Northern Hemisphere experienced virtually no change in spite of all the changes that occurred in the Southern Hemisphere. These results of simulations suggest that the north and the south are linked very weakly and that many of solar cycle characteristics within a hemisphere can be reproduced from information from previous cycles only from that hemisphere (Dikpati et al., 2007).

Our results that the Waldmeier effect is apparently stronger for the SSN of Northern Hemisphere than that for the SSN of Southern Hemisphere may indicate that the dynamo in the two hemispheres is conducted independently as was indicated in simulations by Belucz and Dikpati (2013). And the finding that the Waldmeier effect is significant only when the SSNs of the whole sphere are analyzed may suggest that the effect is not a manifestation of some elementary dynamo process, but it is a phenomenon which appears by a "coarse-grained" overview.

Interestingly, the amplitude of odd-numbered cycles is well correlated with the amplitude of the preceding evennumbered cycles. Figure $4 \mathrm{a}$ and $\mathrm{b}$ are scatterplots between the maximum SSN of even-numbered cycles and that of the preceding odd-numbered cycles, and between the maximum SSN of odd-numbered cycles and that of the preceding even-numbered cycles, respectively. Figure $4 \mathrm{c}$ and $\mathrm{d}$ as well as Fig. 4e and $\mathrm{f}$ are similar scatterplots except that between the successive average SSNs, and between the maximum SSN and the average SSN of the preceding cycle. Although it should be taken care that there are a few outliers, it is seen from Figs. 4b, d, and $\mathrm{f}$ that the amplitude of oddnumbered cycles is excellently correlated with that of the preceding even-numbered cycles, carrying correlation coefficients of $0.972,0.985$ and 0.986 , respectively, when outliers are excepted. The $P$ value in the null hypothesis test for the correlation is as small as $5.386 \times 10^{-5}, 8.282 \times 10^{-6}$ and $7.229 \times 10^{-6}$ for each of the cases shown in Figs. $4 \mathrm{~b}$, d, and f. On the other hand, the amplitude of even-numbered cycles is only weakly correlated with the amplitude of the preceding odd-numbered cycles. Correlation coefficient obtained from the scatterplots shown in Figs. 4a, c, and e is $0.393,0.536$ and 0.436 , and the $P$ value for each case is $0.231,0.089$ and 0.821 , respectively. That is, the null hypothesis that there exists no correlation between the amplitude of even-numbered cycles and that of the preceding odd-numbered cycle is not denied at the confidence level of $5 \%$ for all of these cases. 
(a)

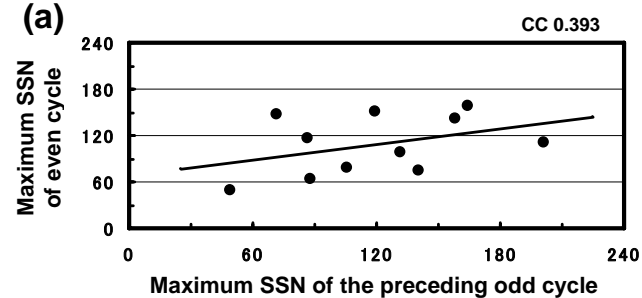

(c)

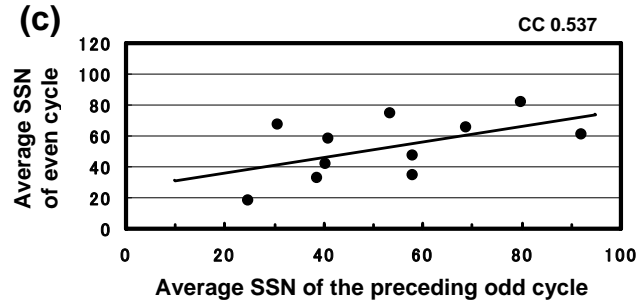

(e)

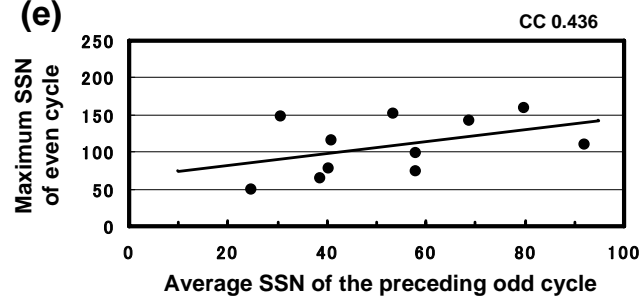

(b)

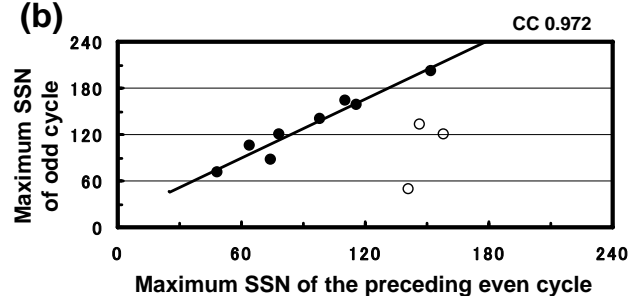

(d)
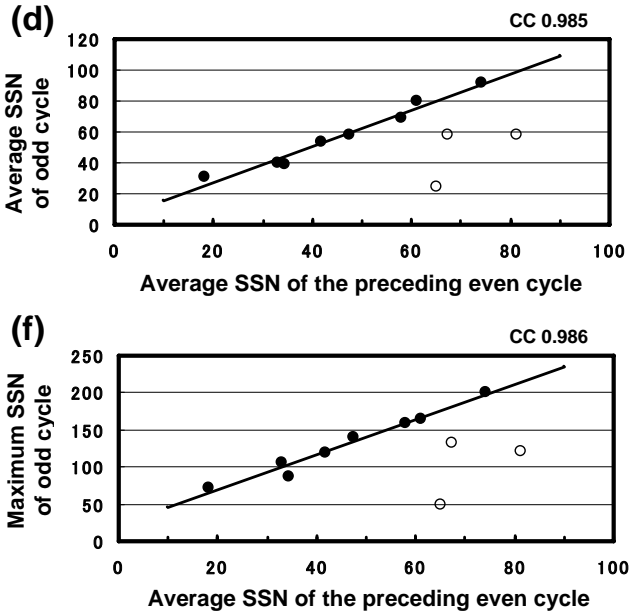

Figure 4. (a) Scatterplot of maximum SSN of even-numbered cycles versus maximum SSN of the preceding odd-numbered cycles. Panel (b) is the same as panel (a) but for odd- and the preceding even-numbered cycles. White circles represent outliers in the correlation. The correlation coefficient and its lower and upper limits of the $95 \%$ confidence interval are $0.393,-0.271$ and 0.803 for the former case, while those for the latter case except three outliers are 0.972, 0.848 and 0.995, respectively. (c) Scatterplot of average SSN of even-numbered cycles versus average SSN of the preceding odd-numbered cycles. Panel (d) is the same as panel (c) but for odd- and the preceding evennumbered cycles. White circles represent outliers in the correlation. The correlation coefficient and its lower and upper limits of the $95 \%$ confidence interval are $0.537,-0.093$ and 0.860 for the former case, while those for the latter case except three outliers are $0.985,0.917$ and 0.997, respectively. (e) Scatterplot of maximum SSN of even-numbered cycles versus average SSN of the preceding odd-numbered cycles. Panel (f) is the same as panel (e) but for odd- and the preceding even-numbered cycles. White circles represent outliers in the correlation. The correlation coefficient and its lower and upper limits of the $95 \%$ confidence interval are $0.436,-0.221$ and 0.821 for the former case, while those for the latter case except three outliers are $0.986,0.920$ and 0.998 , respectively.

We do not know at present the reason why outliers appear in the strong relationship between the amplitude of an evennumbered cycle and that of the succeeding odd-numbered cycle. However, as will be explained in the Discussion, appearance of an outlier is foreseen in the early stage of the preceding even-numbered cycle. Before proceeding to that we try to predict the amplitude of cycle 25 using the above-described correlations.

\section{Prediction of the maximum SSN of cycle 25}

Now that more than 3 years have passed since the SSN took the latest minimum value in December 2008, we can use the excellent relationship between the SSN at point 3 years after the minimum and the average SSN for even-numbered cycles (Fig. 2d) to estimate the average SSN of the current cycle 24 . The estimated value, $39.4 \pm 4$, is plotted with a bar of 1- $\sigma$ error range on the scatterplot between the SSN at a point 3 years after the minimum and the average SSN for even-numbered cycles (Fig. 5a). In order to predict the maximum SSN of cycle 25 , we next use the strong correlation between the maximum SSN of odd-numbered cycles and the average SSN of the preceding even-numbered cycles (Fig. 4f). The predicted value is $115.4 \pm 11.9$ (Fig. 5b). The maximum SSN of cycle 25 can also be estimated by using the relationships shown in Figs. $2 b$ and $4 b$, where a value $116.5 \pm 15.9$ is given.

Although outliers occasionally appear in the relationship between the amplitude of even-numbered cycles and that of the succeeding odd-numbered cycles, we have a good reason to consider that cycle 25 will not be an outlier (see Fig. 6).

A peak of the SSN has been observed in February 2012. If the peak value 66.9 turned out to be the maximum SSN of cycle 24 eventually, the maximum SSN of cycle 25 would be predicted to be $98 \pm 15.9$ by using the correlation between the maximum SSN of an even-numbered cycle and that of the succeeding odd-numbered cycle (Fig. 4b). However, the observed peak value is much smaller than the value of the 
(a)
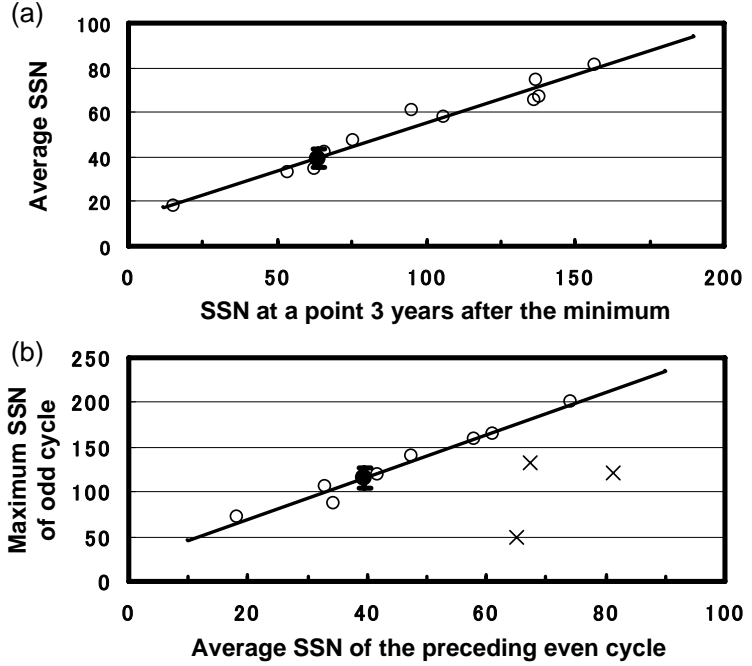

Figure 5. (a) Scatterplot of average SSN versus SSN 3 years after the minimum for even-numbered cycles. The estimated average SSN for cycle 24 is represented by a solid circle with a bar of 1$\sigma$ range. (b) Scatterplot of maximum SSN of odd-numbered cycles versus average SSN of the preceding even-numbered cycles. A solid circle shows the value of the predicted maximum SSN of cycle 25 .

maximum SSN estimated by the correlation shown in Fig. 2b. The difference between the observed peak value and the estimated value of the maximum SSN, 81.3, is 14.4, which amounts to about 2.5 times the standard deviation 5.8 of the excellent correlation between the SSN at a point 3 years after the minimum and the maximum SSN for even-numbered cycles (Fig. 2b). Here, it is worth noting that many cycles showed two or more peaks, and cycles for which a higher peak appeared later are not rare. It has occurred in cycles 5, 12 and 16, for example. Therefore, we think it is highly probable that the real maximum SSN of the current cycle has not yet appeared. In fact, according to the sunspot number data of the Solar Influence Data Analysis Center, a monthly smoothed SSN at September 2013 was 73.1, though the value is still provisional as of April 2014. In any case, we will see if the prediction of the amplitude of the current cycle based on the correlation between even-numbered and the successive odd-numbered cycles is successful or not before long.

\section{Discussion}

In predicting the maximum SSN of cycle 25, we used the strong correlation between the amplitude of even-numbered cycles and that of the succeeding odd-numbered cycles. A problem in making the prediction on the basis of the correlation is that there are outliers in the correlation, as is seen in Fig. 4b, d, and f. At present, we do not know why such apparent outliers appear in the strong correlation. However, we can foresee appearance of an outlier in the early stage of the preceding even-numbered cycle. Figure $6 a$ shows changes in the (a)
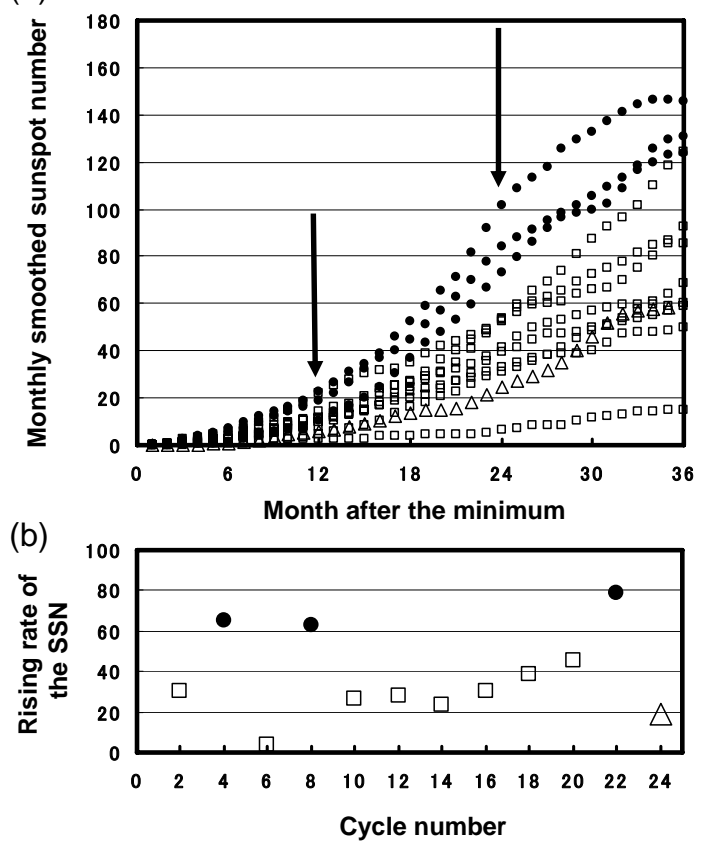

Figure 6. (a) Increase of the SSN of even-numbered cycles in 36 months after taking minimum values. Curves plotted by solid points show cycles 4,8 , and 22 for which succeeding odd-numbered cycles became outliers. Minimum values are set to be 0 for all cycles. (b) Increasing rate of the SSN during the period 12-24 months after minima. Note that the increasing rate for cycles 4,8 , and 22 were exceptionally large. A triangle represents the increasing rate of the current cycle 24. Its small increasing rate suggests that cycle 25 will not be an outlier.

SSN for 12 even-numbered cycles during the first 36 months after taking a minimum value, where cycles 4,8 , and 22 , for which succeeding odd-numbered cycles became outliers, are plotted by solid points. Note that the rate of increase of the SSN in the early stage of cycles 4,8 , and 22 was exceptionally large. That characteristic is obviously seen in Fig. 6b, where the rate of increase during the period 12-24 months after minima is shown. Now, if noted that the rate of increase of the current cycle 24 , shown by a triangle, is such a small value, we may say with certainty that cycle 25 will not be an outlier.

We emphasized in this paper that there exist significant differences in the amplitude-amplitude relationship between pairs of even- and successive odd-numbered cycles and those of odd- and successive even-numbered cycles. At present, we do not know the physical basis that causes the difference. We admit it cannot be denied that the strong correlation between pairs of even- and successive odd-numbered cycles may just be a coincidence due to the limited data of the SSN. The fact that apparent outliers appear in the correlation is actually a problem that cannot be neglected. However, even so, we think the difference in the correlations between evennumbered cycles and odd-numbered cycles is significant. As 
is seen in Fig. 1a and b, the correlation between the SSN at a point 3 years before the minimum and the amplitude of the following cycle is incomparably better for even-numbered cycles than that for odd-numbered cycles. The correlation between the SSN at a point 3 years after the minimum and the maximum SSN of the cycle is also better for even-numbered cycles compared to that for odd-numbered cycles. All of these characteristics seem to indicate that change of the SSN in even-numbered cycles is more regulated than that in oddnumbered cycles, and that the so-called Hale cycle, i.e., the 22-year-period solar cycle which takes a reversal of the sun's dipole magnetic field into account, consists of pairs of an even-numbered cycle and the succeeding odd-numbered cycle, not those of an odd-numbered cycle and the following even-numbered cycle. We speculate that physical process that underlies the Hale cycle might be participating in creating the significant difference in regularities between evennumbered and odd-numbered cycles.

Observation of the correlation between the SSN at a point 3 years before the minimum and the amplitude of the following cycle, especially that for even-numbered cycles, as well as the correlation between amplitudes of even- and successive odd-numbered cycles, may be a sign of the socalled memory effect in solar cycles. By simulating solar cycles using a flux-transport-type dynamo model, Dikpati and Gilman (2006) showed that the combination of poloidal magnetic fields in the past three cycles $(n-1, n-2$, and $n-3)$ contributes in determining the strength of cycle $n$. Based on a similar Babcock-Leighton model, Charbonneau and Dikpati (2000) obtained a result that suggests a correlation with a lag time of two cycles. On the other hand, Yeates et al. (2008), using their Babcock-Leighton-type model, found that when magnetic flux is assumed to be transported dominantly by diffusion, not by advection, the memory persists for mainly one cycle. In any case, existence of an inter-cycle correlation is a basis of the search for "precursors". An extreme idea on this matter is the one presented by Cameron and Shussler (2008), who maintained that most precursors so far proposed can be explained by overlapping of cycles and the Waldmeier effect, though they do not deny the possibility that some memory exists. They noted the importance of finding a quantity that is not "contaminated" by early information leaking in from the new cycle. I do not claim that the correlation shown here corresponds to the one, but I think something meaningful is hidden in the difference between even- and odd-numbered cycles.

It might not be worthless to note here that, besides the positive correlation between the average SSN for evennumbered $(2 n)$ cycles and that for the succeeding oddnumbered $(2 n+1)$ cycles, the average SSN for $(2 n+1)$ cycles is weakly negatively correlated to that for $(2 n+4)$ cycles (Yoshida and Sayre, 2012). It is interesting that when these positive and negative correlations are combined, solar cycles are divided into two series that do not mix or merge, where one is composed of cycles $1,4,5,8,9,12,13,16,17,20,21$, $\ldots$, and the other is composed of $2,3,6,7.10,11,14,15$, $19,23, \ldots$ This finding does not necessarily mean that solar activity could be predicted over a long range, for outliers do appear occasionally in the correlations. Nevertheless, this pattern of inter-cycle relatedness may lead to better understanding of the solar dynamo.

Acknowledgements. The author thanks the SIDC-team, World Data Center for the Sunspot Index, Royal Observatory of Belgium for the smoothed monthly mean SSNs, and the National Astronomical Observatory of Japan for the sunspot data. I thank Leif Svalgaard for reviewing the manuscript and for his helpful comments. I also thank an anonymous reviewer for a number of useful comments that have helped improve the paper.

Topical Editor M. Temmer thanks L. Svalgaard and one anonymous referee for their help in evaluating this paper.

\section{References}

Belucz, B. and Dikpati, M.: Role of asymmetric meridional circulation in producing north-south asymmetry in a solar cycle dynamo model, Astrophys. J., 779, doi:10.1088/0004637X/779/1/4, 2013.

Cameron, R. and Schussler, M.: Solar cycle prediction using precursors and flux transport models, Astrophys. J., 659, 801-811, 2007a.

Cameron, R. and Schussler, M.: Are solar cycle predictable?, Astron. Nachr, 328, 1087-1091, 2007b.

Cameron, R. and Schussler, M.: A robust correlation between growth rate and amplitude of solar cycles: Consequences for prediction methods, Astrophys. J., 685, 1291-1296, 2008.

Charbonneau, P. and Dikpati, M.: Stochastic fluctuations in a Babcock-Leighton model of the solar cycle, Astrophys. J., 543, 1027-1043, 2000.

Dikpati, M. and Gilman, P. A.: Simulating and predicting solar cycles using a flux-transport dynamo, Astrophys. J., 649, 498-514, 2006.

Dikpati, M., de Toma, G., and Gilman, P. A.: Predicting the strength of solar cycle 24 using a flux-transport dynamo-based tool, Geophys. Res. Lett., 23, L05102, doi:10.1029/2005GL025221, 2006.

Dikpati, M., Gilman, P. A., de Toma, G., and Ghosh, S. S.: Simulating solar cycles in northern and southern hemisphere by assimilatingmagnetic data into a calibrated flux-transport dynamo, Solar Physics, 245, 1-17, 2007.

Dikpati, M., Gilman, P. A., and de Toma, G.: The Waldmeier effect: An artifact of the definition of Wolf sunspot number?, Astrophys. J., 673, L99-L101, 2008.

Hathaway, D. H. and Wilson, R. M.: Geomagnetic activity indicates large amplitude for sunspot cycle 24, Geophys. Res. Lett., 33, L18101, doi:10.1029/2006GL027053, 2006.

Hathaway, D. H., Nandy, D., Wilson, R. M., and Reichmann, E. J.: Evidence that a deep meridional flow sets the sunspot cycle period, Astrophys. J., 589, 665-670, 2003.

Hathaway, D. H., Nandy, D., Wilson, R. M., and Reichmann, E. J.: Erratum: "Evidence that a deep meridional flow sets the sunspot cycle period, Astrophys. J., 602, p. 543, 2004. 
Kane, R. P.: A preliminary estimate of the size of the coming solar cycle 24, based on Ohl's precursor method, Solar Phys., 243, 205-217, 2007.

Ohl, A. I.: Forecast of sunspot maximum number of cycle 20, Solice Donie, 9, 84-85, 1966.

Pesnell, W. D.: Prediction of solar cycle 24, Solar Phys., 252, 209_ 220, 2008.

Schatten, K. H.: Fair space weather for solar cycle 24, Geophys. Res. Lett., 32, L21106, doi:10.1029/2005GL024363, 2005.

Svalgaard, L., Cliver, E. W., and Kamide, Y.: Sunspot cycle 24: Smallest cycle in 100 years?, Geophys. Res. Lett., 32, L01104, doi:10.1029/2004GL021664, 2005.

Thompson, R. J.: The rise of solar cycle number 22, Solar Phys., 117, 279-289, 1988.
Waldmeier, M.: Neue Eigenschaften der Sonnenfleckenkurve, Astronomische Mitteilungen Zurich, 14, 105-130, 1935.

Yeates, A. P., Nandy, D., and Mackay, D. H.: Exploring the physical basis of solar cycle predictions: Flux transport dynamics and presence of memory in advection- versus diffusion-dominated solar convection zones, Astrophys. J., 673, 544-556, 2008.

Yoshida, A. and Sayre, R.: Tendency of discreteness of the solar amplitude and intercycle relatedness, Advances in Astronomy, 2012, 519852, doi:10.1155/2012/519852, 2012.

Yoshida, A. and Yamagishi, H.: Predicting amplitude of solar cycle 24 based on a new precursor method, Ann. Geophys., 28, 417425, doi:10.5194/angeo-28-417-2010, 2010. 